\title{
Intimal Sarcoma of the Descending Aorta Mimicking Aortitis
}

Angela Pucci, MD, $\mathrm{PhD}^{1}$, Andrea De Martino, $\mathrm{MD}^{2}$, Maurizio Levantino, $\mathrm{MD}^{2}$, Raffaella Berchiolli, MD², Fulvio Basolo, MD', Uberto Bortolotti, MD²*

${ }^{1}$ Division of Pathology, University Hospital, Pisa, Italy

2 Division of Cardiac Surgery, University Hospital, Pisa, Italy

${ }^{3}$ Division of Vascular Surgery, University Hospital, Pisa, Italy

\begin{abstract}
We describe a 74-year-old male patient with an intimal sarcoma of the descending aorta mimicking aortitis. The patient presented with lower back pain, fever, and increased C-reactive protein, erythrocyte sedimentation rate, and immunoglobulin G4 (IgG4) serum levels, together with Staphylococcus epidermidis-positive blood cultures. These findings, together with evidence of a 49-mm pseudoaneurysm of the descending thoracic aorta, caused us to suspect aortitis. However, postoperative histology and immunohistochemistry demonstrated the presence of an intimal aortic sarcoma. At the 8-month follow-up, local recurrence of the neoplasm and lung metastases were noted.

Copyright $\odot 2016$ Science International Corp.
\end{abstract}

\section{Key Words}

Aorta • Aortitis • Aortic tumor

\section{Introduction}

Intimal sarcomas are extremely uncommon tumors involving the major vessels, mostly the pulmonary artery and the aorta, with a dismal prognosis [1]. The clinical presentation is usually nonspecific and mostly related to tumor embolism resulting in limb or visceral ischemia [1, 2]. Preoperative diagnosis is difficult and most neoplasms are recognized only upon histological analysis of the surgical specimen. Here, we describe an unusual case of intimal sarcoma of the descending thoracic aorta mimicking aortitis.

Published by Science International Corp. ISSN 2325-4637

Fax +1 2037853552

E-Mail: aorta@scienceinternational.org

http://aorta.scienceinternational.org
Accessible online at:

http://aorta.scienceinternational.org

\section{Case Presentation}

A 74-year-old male was admitted to our department complaining of abdominal and lower back pain and fever for a duration of three months. The patient had also previously been diagnosed with hypertension, hypercholesterolemia, stage 3 chronic kidney disease, and monoclonal gammopathy of unknown significance. Laboratory tests revealed increased erythrocyte sedimentation rate and serum levels of Creactive protein and IgG4 with mild anemia. Blood cultures were positive for Staphylococcus epidermidis. Computed tomography (CT) with contrast-enhanced angiography showed a disruption of the anterior wall of the aorta with a maximum diameter of $49 \mathrm{~mm}$ surrounded by contrast enhancing solid tissue at the thoraco-abdominal transition and close to the origin of the celiac trunk, which was interpreted as a pseudoaneurysm (Figure 1A and 1B). To better define the aortic lesion, ${ }^{18} \mathrm{~F}$-fluorodeoxyglucose (FDG) positron emission tomography (PET) was performed, revealing isolated localization of the radionuclide at the level of the pseudoaneurysm (Figure 1C and 1D).

A differential diagnosis between infective aortitis and aortitis associated with lgG4-related sclerosing disease was considered. Accordingly, steroid and antibiotic therapy was started with no clinical improvement or improvement of laboratory test findings after 10 days.

The patient underwent surgical resection of the pseudoaneurysm through a left thoracotomy in the

\footnotetext{
* Corresponding Author:

Uberto Bortolotti, MD

University Hospital

Division of Cardiac Surgery

Via Paradisa, 2, 56124 Pisa, Italy

Tel.: +1 3950 995250; Fax: +1 3950 541647; E-Mail: uberto.bortolotti@med.unipi.it
} 

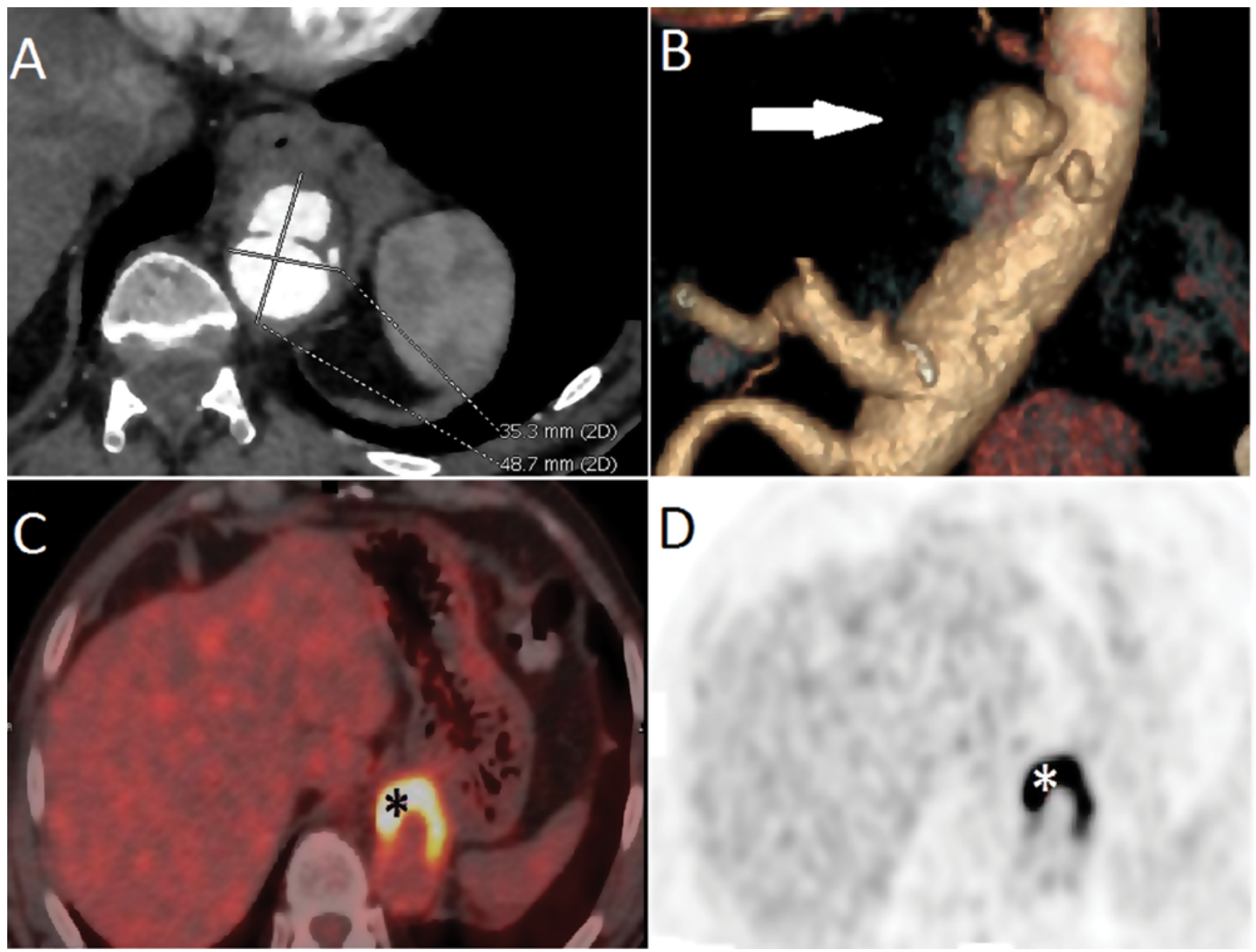

Figure 1. Panel A. Angio-computed tomography (CT) showing the pseudoaneurysm in the descending thoracic aorta. Panel $B$. Volume-rendered image showing the close relation of the pseudoaneurysm with the celiac trunk (arrow). Panels C and D. CT positron emission tomography demonstrating intense tracer uptake in the aortic wall.

7th intercostal space combined with a laparotomy and a retroperitoneal approach. The operation was performed with left heart bypass by cannulating the left femoral artery and the left inferior pulmonary vein under moderate hypothermia $\left(32^{\circ} \mathrm{C}\right)$. The pseudoaneurysm was isolated and transected and the aorta was reconstructed with the interposition of a 24-mm cryopreserved homograft. The postoperative course was uncomplicated and the patient was discharged home on the 8th postoperative day.

Both histology and microbiological examinations were performed on the resected aortic tissue. By routine histology and immunohistochemistry, an intimal aortic sarcoma was diagnosed (Figure 2). The aortic wall was extensively infiltrated by a poorly-differentiated spindle cell tumor with nuclear pleomorphism, abundant mitoses, and microfoci of necrosis originating from the intimal surface and infiltrating the aortic wall including the resected margins. Immunohistochemistry, performed using an automated immunostainer and specific antibodies (Ventana Medical Systems, Inc., Tucson, AZ, USA), showed diffuse staining for vimentin andfocal Mouse double minute 2 homolog (MDM2) positivity. Markers for epithelial cells, smooth and skeletal muscle cells, leukocytes, and melanocytes were otherwise negative.

The patient was treated with a specific protocol of chemotherapy with close clinical follow-up. However, the tumor relapsed soon after surgery with develop- 


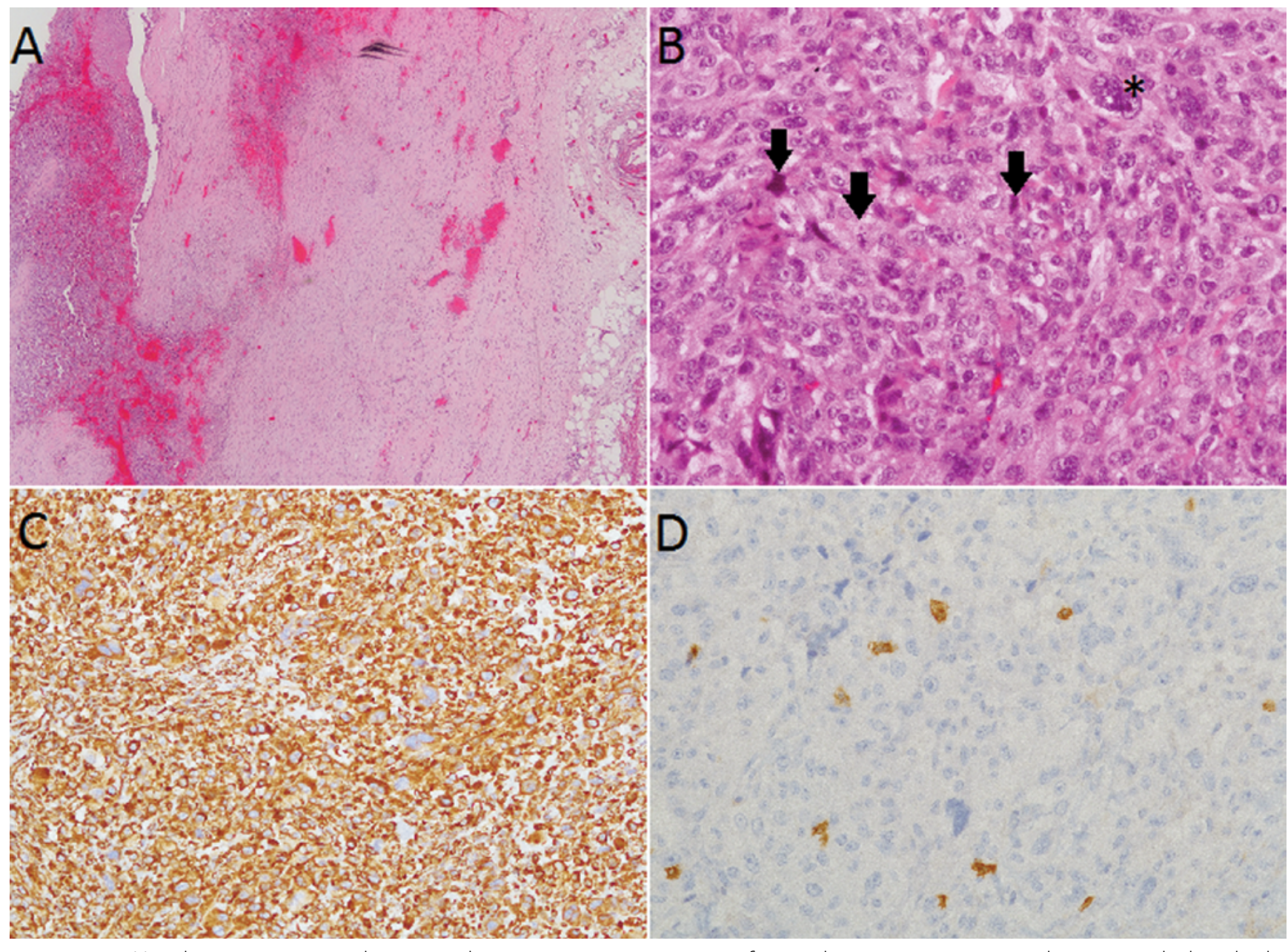

Figure 2. Histologic sections showing the sarcoma originating from the aortic intima, characterized by highly atypical cells with nuclear pleomorphism (asterisk) and mitoses (arrows), and diffuse immunoreactivity for vimentin and focal positivity for mouse double minute 2 homolog (MDM2). Panel A. Haematoxylin and eosin stain, original magnification 2x. Panel B. Haematoxylin and eosin stain, original magnification 20x; Panel C. Immunoperoxidase staining and haematoxylin counterstaining using a specific antibody against Vimentin, original magnification 10x; Panel D. Immunoperoxidase staining and haematoxylin counterstaining using a specific antibody against MDM2, original magnification 20x.

ment of local recurrence and lung metastases at 8-month follow-up.

\section{Discussion}

The present case is, to our knowledge, the first reported aortic intimal sarcoma mimicking aortitis and presenting as a descending aortic pseudoaneurysm. Intimal aortic sarcomas are extremely rare highgrade malignant neoplasms of large blood vessels, sub-classified into intimal tumors characterized mainly by intraluminal growth, mural tumors of medial or adventitial origin, and mixed tumors [3]. Such neo- plasms are poorly differentiated and usually have a nonspecific presentation. The differential diagnosis requires histological analysis demonstrating a malignant mesenchymal tumor, and immunohistochemistry revealing immunoreactivity only for vimentin and MDM2. Vimentin is a sensitive marker for many neoplasms, while MDM2 is found in over $70 \%$ of intimal sarcomas and is a negative prognostic marker. Indeed, most cases have been diagnosed either at necropsy or at examination of aortic surgical specimens [1, 2]. An intimal sarcoma must be differentiated from a more common angiosarcoma, which shows definite and diffuse endothelial differentiation, and from a ley- 
omiosarcoma, which is characterized by smooth muscle marker immunoreactivity [4-6]. The pathogenesis of intimal sarcoma is still unknown, but genetic factors, including changes in the MDM2-p53pathway, seem to play important roles [7].

Intimal aortic sarcomas are usually localized in the abdominal aorta between the celiac artery and the iliac bifurcation, and in $30 \%$ of cases the descending aorta is involved as seen in the present case $[1,2,4,6]$. The most common clinical presentation is related to tumor embolization with peripheral or mesenteric ischemia and necrotic skin lesions, or to metastasis, which occurs mainly to bones, liver, and lungs. Less common symptoms are secondary to intraluminal occlusion of the aorta, mass effect, or pseudoaneurysm formation with or without secondary aortic rupture [6].

Imaging studies of the aorta, such as a CT scan, can seldom distinguish aortic intimal tumors from advanced atherosclerotic disease. Even ${ }^{18} \mathrm{~F}$-FDG PET may not specifically differentiate a tumor from an infectious aortic lesion as observed in the present case. This has been emphasized by Naughton et al. [6] who reported a patient with an aortic neoplasm presenting as an abdominal aortic rupture with clinical and radiologic findings suggestive of aortitis. In tumors of the pulmonary artery, the presence of intraluminal filling defect enhancement on MRI with gadolinium has been shown to be the most sensitive diagnostic tool to differentiate such tumors from non-neoplastic lesions [8]; it can be speculated that this finding could also be applied to aortic lesions.

Primary malignant tumors of the aorta have a poor prognosis, with a mean survival time of 14 months [6]. The most effective treatment is surgical resection with graft interposition, but radical surgery is often not feasible because of either tumor volume or local extension with adjacent tissue infiltration. In the present case, operation consisted of excision of the pseudoaneurysm and reconstruction of the aorta by interposition of a homograft because of the suspicion of an infective nature of the lesion. Postoperative histological and immunoistochemical analyses ruled out the presence of aortitis, revealing instead a rare malignant neoplasm.

In conclusion, intimal sarcomas of the aorta are rare and aggressive neoplasms which occasionally may mimic aortitis. The preoperative diagnosis appears of utmost importance because an unsuspected malignancy, as in the case herein reported, may lead to incomplete wide margin resection, thus influencing the long-term prognosis. Indeed, our patient has shown tumor recurrence at 8-month follow-up.

\section{Conflict of Interest}

The authors have no conflict of interest relevant to this publication.

\section{Comment on this Article or Ask a Question}

\section{References}

1. Bode-Lesnievska B, Debiec-Rychter M. Intimal sarcoma. In: Fletcher CDM, Bridge JA, Hogendoorn PCW, Mertens F. WHO classification of tumors of soft tissue and bone, $4^{\text {th }}$ Edition. Lyon: IARC; 2013, p. 232-233.

2. Sebenik M, Ricci A Jr, DiPasquale B, Mody $K$, Pytel $P$, Jee $K J$, et al. Undifferentiated intimal sarcoma of large systemic blood vessels: report of 14 cases with immunohistochemical profile and review of literature. Am J Surg Pathol. 2005;29:1184-1193. DOI: 10.1097/01.pas.0000159774.70288.7d

3. Wright EP, Glick AD, Virmani R, Page DL. Aortic intimal sarcoma with embolic metastases. Am J Surg Pathol. 1985;9:890897. DOI: 10.1097/00000478-19851200000005

4. Staats P, Tavora F, Burke AP. Intimal sarco- mas of the aorta and iliofemoral arteries: a clinicopathological study of 26 cases. Pathology. 2014;46:596-603. DOI: 10.1097/ PAT.0000000000000182

5. Lazar A, Evans HL, Shipley J. Leiomyosarcoma. In: Fletcher CDM, Bridge JA, Hogendoorn PCW, Mertens F. WHO classification of tumors of soft tissue and bone, $4^{\text {th }}$ Edition. Lyon: IARC; 2013, p. 111-113.

6. Naughton PA, Wandling $M$, Phade $S$, Garcia-Toca M, Carr JC, Rodriguez HE. Intimal angiosarcoma causing abdominal aortic rupture. J Vasc Surg. 2011;53:818-821. DOI: 10.1016/j.jvs.2010.10.090

7. Zhang $H$, MacDonald WD, EricksonJohnson $M$, Wang $X$, Jenkins RB, Oliveira AM. Cytogenetic and molecular cytogenetic findings of intimal sarcoma. Cancer
Genet Cytogenet. 2007;179:146-149. DOI: 10.1016/j.cancergencyto.2007.08.013

8. Restrepo CS, Betancourt SL, Martinez-Jimenez S, Gutierrez FR. Tumors of the pulmonary artery and veins. Semin Ultrasound CT MR. 2012;33:265-272. DOI: 10.1053/j.sult.2012.04.002

Cite this article as: Pucci $A$, DeMartino A, Levantino $M$, Berchiolli $R$, Basolo $F$, Bortolotti U. Intimal Sarcoma of the Descending Aorta Mimicking Aortitis. AORTA (Stamford) 2016;4(4):142-145. DOI: $\quad$ http://dx.doi.org/10.12945/j. aorta.2016.16.022 